\title{
THE ALGORITHMS OF QUASI-OPTIMAL PICTURE AREAS MATCHING
}

\author{
A.A. Doudkin ${ }^{1)}$, R.Kh. Sadykhov ${ }^{2)}$, M.E. Vatkin ${ }^{1)}$ \\ 1) United Institute of Informatics Problems of NASB, 6, Surganova str., BY-220012, Minsk, Belarus, \\ doudkin@newman.bas-net.by, vatkin@1si.bas-net.by, http://lsi.bas-net.by/ \\ 2) Belarusian State University of Informatics and Radioelectronics, Brovka str. 6, Minsk, 220027, Belarus, \\ rsadykhov@gw.bsuir.unibel.by
}

\begin{abstract}
Using common matching quality criteria the problem of optimum matching of partially overlapped picture areas is considered. Two schemes of algorithms are proposed for quasi-optimal solution of the problem with following restrictions: the areas are rectangular and have an identical scale. In the first scheme local criterion is used to estimate a quads of frames located in square matrix. In the second scheme is used the special distance function based on neighbor picture matching criterion. The algorithms are realized in program system of layout metallization restoring of integrated circuits.
\end{abstract}

Keywords: - Image matching, similarity function, searching algorithm, integrated circuit layer

\section{INTRODUCTION}

The most of big images are an assembly of some picture areas. The neighboring picture areas have overlapping regions. That's way a combine of the partially overlapping picture areas into one image is one of main task of image registration methods [1].

The main principle of the picture areas matching is based on correlation of the areas in the overlapping region. There are two approaches to extending correlation to include wider ranges of distortions. One method is to successively distort and correlate a reference template across a full range of distortions [2]. Classical technique of correlation and relaxation is used to find an initial set of matches, then a robust technique of the Least Median of Squares is used to discard false matches in this set. It is computationally very expensive. The algorithm calculates the squared difference per pixel of all possible overlaps between two adjacent images and selects the minimum. Using special transformations of initial images it is possible to speed up correlation.

Another approach bases on composite filtering, where a single filter responds to a full range of distortions [3]. Good technique for matching involves Fourier autocorrelation [4]. This technique replaces convolution-based template matching by Fourier transforming the source and template images, multiplying one by the complex conjugate of the other, then inverse Fourier transforming the result. The result should ideally provide a single peak at the single point of correlation.
Another method uses parts of overlapping regions that in sum is smaller then initial overlapping region [5-6]. For our application it is difficult to use this approach.

Within this approaches it is possible to use parallel matching algorithm to speed up a calculation process. First some adjacent picture areas are selected. Then, it iterates over all possible overlaps of these areas calculates (in parallel) the sum of the differences between each pixel in the overlapping regions.

The main attention is paid to strategies for finding near-optimal matching algorithms. They are very important to reduce a computational complexity of matching. In [8] the following search algorithm was proposed. The search starts with the image in the lower-left corner of the matrix. It adds this image to the used frames set. From here it finds the image closest to the lower-left image that does not exist in the used frames set and enters a loop over all possible ranges of $\mathrm{x}$ and $\mathrm{y}$ motion (these ranges are user-selectable). At every possible shift of the image, it calculates the overlap area with each of the images in the used frame set and, if this area exists, calculates the root mean squared correlation error in the overlap region. If the error is less than the current minimum, the position is noted and the minimum value is updated. In this way, the minimum correlation of the image against all overlapping, previously matched images is determined. Once the entire range is evaluated, that image is moved to the position yielding the 
minimum correlation and it is added to the list of used frames. The process then repeats with the next image and continues until all images have been matched.

We discuss a matching problem in following two applications: wafer production and printed circuit boards inspection. The picture areas are calibrated i.e. with known intrinsic parameters (have the same value of pixels). They are only rectangle images (Fig. 1), there are no rotations, that's way any overlapping regions are rectangles too.

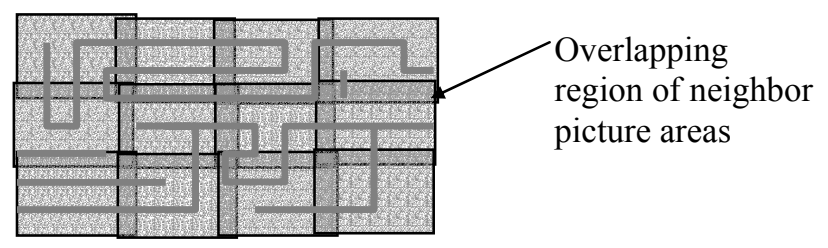

Fig. 1 - Image of fragment of IC metalization layer.

The images have been taken by a single camera but at different time instants or different light. There are some misplacement due to effect of perspective distortion or blurring occurs when object is out of focus on boundaries or object is vary in height.

Here we describe algorithms those allow to reduce a time complexity of matching. The optimization-matching problem is formulated by the use common criteria of matching. Two schemes of algorithms are proposed, those give us near optimal solutions for frames matching. Three or four fragments matching is used instead of known algorithms to obtain good matching.

\section{PROBLEM DEFINITION}

We define a matching problem as follows.

An initial data for matching is the matrix $\left[P_{i, j}\right]$ of the partially overlapped picture areas, $i=\overline{1 \ldots n}$, $j=\overline{1 . . . m}$.

Let's consider undirected graph $G=(N, R)$ that sets up an adjunct relation of the picture areas, where $N$ - set of nodes corresponding to the picture areas and $R$ - set of edges corresponding to the adjacent picture areas. For easiness, we can consider only vertical or horizontal adjacent. For the initial matrix of dimensionality $\mathrm{n} \times m$ we have $N=\left\{\mathrm{n}_{\mathrm{i}, \mathrm{j}}\right.$, $i=\overline{1 . . n}, j=\overline{1 \ldots m}\}, R=\left\{r_{k}, k=1,2, \ldots, 2 m n-m-n\right\}$.

Let's assign $S_{\mathrm{g}}$ and $\mathrm{S}_{\mathrm{v}}$ - the sizes of overlapping regions on horizontal and vertical adjacent accordingly and $\Delta \mathrm{g}, \Delta \mathrm{v}$ are the possible horizontal and vertical deviation of the overlapping regions.

The similarity function of two picture areas $P_{l}$ and $P_{2}$ is the discrete distance function $d\left(P_{1}, P_{2}, h, v\right)$ where $h$ and $v$ are horizontal and vertical shifts of the picture area $P_{2}$ relative to the picture area $P_{1}$ accordingly, $-\Delta h \leq h \leq \Delta h,-\Delta v \leq v \leq \Delta v$. For convenience we shall write $d\left(r_{i}, h, v\right)=d\left(P_{1}, P_{2}, h\right.$, $v)$ where $r_{i}$ - is the edge of graph $G$ that defines proximity relation of $P_{1}$ and $P_{2}$.

Let's assume that the best position of two neighbor picture areas (relative to each other) is the position where $d$ has minimal value.

So the goal function is defined in the following way:

$$
g=\sum_{i=1}^{2 m n-m-n} d\left(r_{i}, h, v\right) \rightarrow \min
$$

The problem is to find the relative coordinates of the picture areas so as the value of the function (1) is minimal.

The trivial solution is the full search of all possible combinations of the overlapping. This is not acceptable because of big amount of the combinations $(2 \Delta)^{2(n m-1)}$. From this point of view the algorithms of restricted search are more preferable if they give user acceptable results.

Two ways of the picture areas matching are considered in the paper. The first way bases on using of the local matching quality criterion g' instead of g. The criterion g' calculates the matching quality of the partial matrix of the graph of the $2 \times 2$ size. In this case the algorithm calculates the best position of four picture areas. These positions are fixed and the algorithm tries to add next two ore three picture areas to the previous ones depending on an algorithm of exhaustive search of the picture areas. Thus the new four picture areas are estimated into which two or one picture areas included from previous four picture areas.

In the second scheme function $\mathrm{g}$ based on local estimation function of overlapping of an picture area with all its adjacent picture areas is used.

The second algorithm uses the function $g$ that is the linear combination of local matching functions. The local matching function defines the matching quality criterion of the picture with its neighbors.

The algorithms are given below.

\section{ALGORITHMS BASED ON 4-AREAS MATCHING FUNCTION}

The basic procedure of fist algorithm is matching of four picture areas.

Step0. Begin.

Given $\left[P_{i, j}\right], G, d$.

Set $\mathrm{i}=0, d_{\text {Opt }},=-1$.

Step 1. 
The initial position of the picture areas $P_{0}, P_{1}, P_{2}$, $P_{3}$ are set in a such way to ensure the average overlapping equal to $S_{h}$ and $S_{v}$ on horizontal and vertical positions respectively.

If $d_{O p t} \geq 0$, go to step 4 .

Step 2.

Calculate the value of function $d$ and set $d_{O p t}=d$, the coordinates of $P_{1}, P_{2}, P_{3}$ is set to $h_{1 O p t}=h_{2 O p t}=$ $h_{3 O p t}=0, v_{1 O p t}=v_{2 O p t}=v_{3 O p t}=0$.

Step 3.

For each picture $P_{1}, P_{2}, P_{3}$ all possible vertical and horizontal shifts by 1 pixel are considered and according value $d_{\mathrm{i}}$ of function is calculated. If $d_{\mathrm{i}}<$ $d_{O p t}$, then $d_{O p t}=d_{\mathrm{i}}, h_{\mathrm{i} O p t}$ and $v_{\mathrm{i} O p t}$, are set to current value of $h_{\mathrm{i}}$ and $v_{\mathrm{i}}, i=1,2,3$.

Repeat step 3 while $d_{O p t}$ is decreasing and $h_{i}<\Delta h$ and $v_{i}<\Delta v$.

Go to step 1.

\section{Step 4.}

For each picture $P_{1}, P_{2}, P_{3}$ all possible vertical and horizontal shifts by -1 pixel are considered and according value $d_{\mathrm{i}}$ of function is calculated. If $d_{\mathrm{i}}<$ $d_{O p t}$, then $d_{O p t}=d_{\mathrm{i}}, h_{\mathrm{i} O p t}$ and $v_{\mathrm{i} O p t}$, are set to current value of $h_{\mathrm{i}}$ and $v_{\mathrm{i}} ., i=1,2,3$.

Repeat step 4 while $d_{O p t}$ is decreasing and $\left|h_{i}\right|<\Delta h$ and $\left|v_{i}\right|<\Delta v$.

\section{Step 5. End.}

The variation of the algorithms differs by the way of new pictures addition. The varieties of algorithms differ by an order of a choice of quads and order of fixing of frames. The fixing means an immovability of frames. The examples illustrating an order of a combination of quads, are shown on Fig. 2 and Fig.3, where the order is indicated in figures. The order of exhaustive search of quads essentially influences quality of a gluing together.

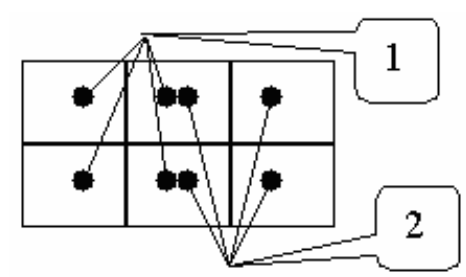

Fig. 2 - Horizontal matching by 2 pucture areas addition.

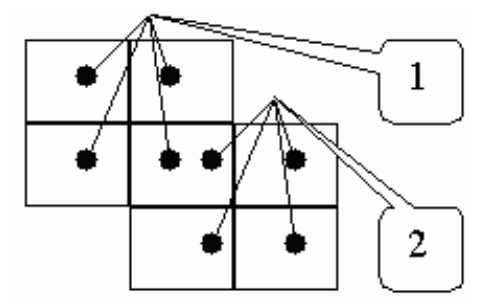

Fig. 3 - Matching by 3 picture areas addition.

\section{SECOND MATCHING ALGORITHM}

Let's define the discrete function $f_{i, j}(x, y)$ that is the distance function between the current position $(x$, $y$ ) of the picture area $P_{i, j}$ and its optimal position regarding to the neighboring picture areas that are fixed:

$f_{i, j}(x, y)=\sum_{k=1}^{K_{i, j}}\left(\left|x-O p t X_{i, j}^{k}\right|+\left|y-O p t Y_{i, j}^{k}\right|\right)$,

where $K_{i, j}$ is the number of the neighboring picture areas of $P_{i, j},(x, y)$ are the current coordinates of $P_{i, j}$, $\left(\right.$ Opt $\left.X_{i, j}^{k}, O p t Y_{i, j}^{k}\right)$ are the optimal coordinates of $P_{i, j}$ and its $k$ neighbor picture areas are in the position where the value of distance function $d$ between $P_{i, j}$ and its $k$ neighbor picture is minimal.

The value function $g$ of picture area matching is defined in the following way:

$$
g=\sum_{i=1, j=1}^{n, m} f_{i, j}(x, y)
$$

where $\mathrm{n} \times m$ is dimensionality of the matrix $\left[P_{i, j}\right]$.

The algorithm minimizes the function (2):

Step0. Begin.

Given $\left[P_{i, j}\right] G, g$.

Set the areas so, that overlapping regions have the sizes $S_{\mathrm{h}}$ for horizontal overlapping and $S_{\mathrm{v}}$ for vertical overlapping.

Set the acceptable error lever Err.

Step 1.

While $\forall i, j \max \left(f_{i, j}\right)>\operatorname{Err}$,

make the shift $\vec{s}_{i, j}$ for the picture area $P_{i, j}$, where

$$
\vec{s}_{i, j}=\left(\sum_{k=1}^{K_{i, j}}\left(x-O p t X_{i, j}^{k}\right), \sum_{k=1}^{K_{i, j}}\left(y-O p t Y_{i, j}^{k}\right)\right) .
$$

Step 2. End.

\section{SYSTEM OF MATCHING}

The matching algorithms are included in the system of processing of layers of integrated circuits $[9,10]$. The scenario of the system has the next stages:

1. Algorithm adjustment.

Set up the values of an average overlap of adjacent picture areas on horizontal and vertical $\left(S_{g}\right.$ and $\mathrm{S}_{\mathrm{v}}$ ) and the values of the possible horizontal and 
vertical deviation of the overlapping regions $(\Delta \mathrm{g}$ and $\Delta \mathrm{v})$.

2. Choice of the matching function and the order of the matching.

Set up a distance function of overlapped frames and a type of searching for matching algorithm (in columns, in rows, block-by-block with the different search schemes using first or second type of matching algorithm.

3. Data initialisation for selected frames.

The matrices are created for each of overlapping pare of picture areas. Each element of the matrix corresponds to a value of distance function between overlapped frames for their different positions. Using criterion of minimum distance the optimal position is found.

4. Calculation of the optimal coordinates using matching algorithm.

The traversal of positioned frames is fulfilled according to matching algorithm and search scheme. After a positioning of all frames, a maximal vector of all produced moving which value is displayed in the main dialog window in current value monitor group are found. The matching is produced repeatedly so long as vector of maximal moving does not become equal to 0 or smaller then user determinates value.

The example of the metallization IC layer picture $\mathrm{m} \_00 . b m p, \mathrm{~m}_{-} 01 . \mathrm{bmp}, \mathrm{m} \_10 . \mathrm{bmp}$ and $\mathrm{m}_{-}$11.bmp is shown in the Fig. 4. Numbers after underlining sign are the indexes of the areas in the matrix. It is clear that matching of the areas with average overlapping $S_{\mathrm{v}}=50$ and $S_{\mathrm{h}}=25$ is not the best solution.

$$
\begin{array}{ll}
\mathrm{m} \_00 . b m p & \mathrm{~m} \_01 . b m p \\
\mathrm{~m}_{-} \text {10.bmp } & \mathrm{m} \_11 . \mathrm{bmp}
\end{array}
$$

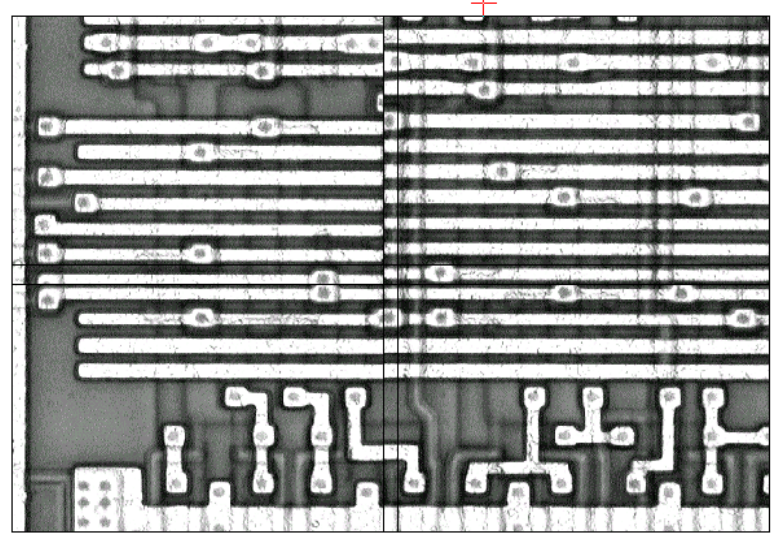

Fig.4 - The matrix of matching areas.

Let's estimate the computational costs of matching algorithms for Celeron-400 and initial data $\left(S_{h}=25, S_{v}=50, \Delta h=10 . \Delta v=40\right)$.

The costs of second algorithm are 1 minute for calculating of the neighbour pictures matching matrixes and 2 seconds for matching step. The matching step is performed by 4 iteration.
The results are shown in the fig.5.
m_00.bmp
m_01.bmp
$\mathrm{m}_{-}-10 . \mathrm{bmp}$
$\mathrm{m}_{-}^{-} 11 . \mathrm{bmp}$

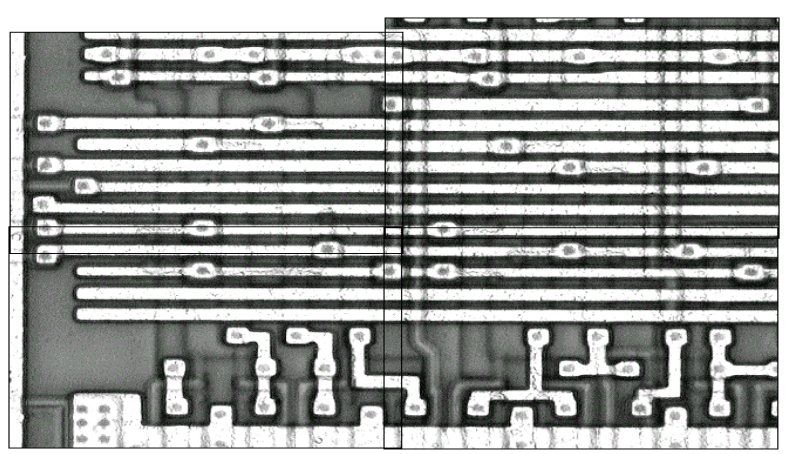

Fig.5 - The matrix after second algorithm matching.

The absolute coordinates of areas are below:

$\begin{array}{lll}25 & 36 & \text { m_00.bmp } \\ 27 & 424 & \text { m_01.bmp } \\ 635 & 34 & \text { m_10.bmp } \\ 637 & 453 & \text { m_11.bmp }\end{array}$

It is clear that results can be improved by shifting of all pictures so that coordinates of first picture will be $(0,0)$.
$\begin{array}{lll}0 & 0 & \text { m_00.bmp }\end{array}$
$2388 \quad$ m_01.bmp
$610-2 \quad \mathrm{~m}_{-}$10.bmp
$612417 \quad \mathrm{~m}-11 . \mathrm{bmp}$

The first algorithm takes 20 minutes of performance time. The matching quality is better but its results are worse in the case when the areas are highly mismatched. It is because of search is finished when first local minimum is found.
m_00.bmp
$\mathrm{m}_{-} 10 . \mathrm{bmp}$
m_01.bmp
m_11.bmp

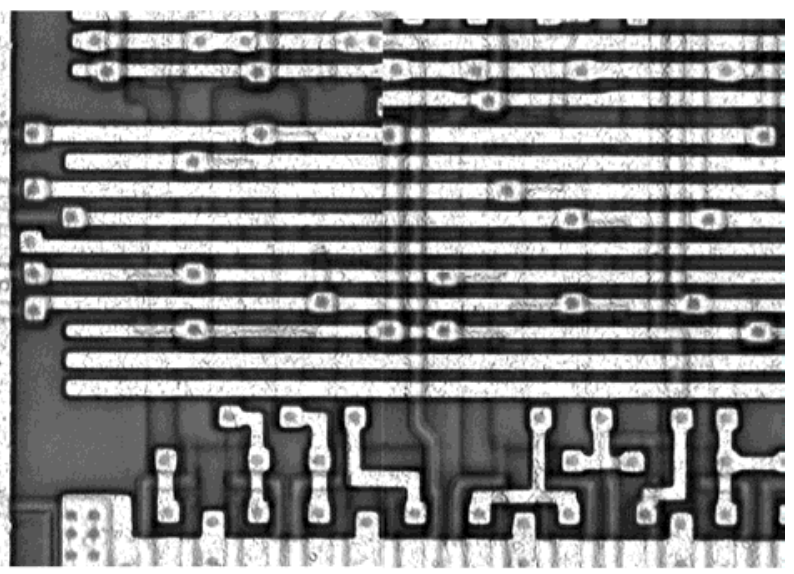

Fig.6. - The example of bad results for first algorithm.

The experiments show that for second algorithm getting into a loop is possible because impossibility of reaching maximal used shift smaller than Err. The pictures used in this case have a chain of minimums 
in the matching matrixes that are approximately equal.

\section{CONCLUSION}

Our algorithms have the following advantage in application to processing of digital video images of topological layouts of an integrated circuit represented as a set of overlapped frames: there are no complex computation needed to perform the mapping. Our experimental results demonstrate the possibility of automatically identifying adjacent positions by maching frames of ICs layouts.

The choice of the type of algorithm depends on overlapping pictures mismatching characteristics. Given variants of algorithms are ordered by requirement of input device accuracy.Research supported in part by Belarussian Foundation Research Fund grant T00- 068.

\section{REFERENCES}

[1] L. G. Brown. A survey of image registration techniques, ACM (Assoc. Comput. Mach.) Comput. Surv. 24, (1992), pp. 325-376.

[2] Z. Zhang, R. Deriche, O. Faugeras and Q.-T. Luong. A Robust Technique for Matching Two Uncalibrated Images Through the Recovery of the Unknown Epipolar Geometry, Artificial Intelligence Journal, 78, (Oct. 1995), pp. 87119.

[3] William J. Chimitt, Jr., and Laurence G. Hassebrook. Scene recons ruction from partially overlapping images with use of composite, $J$. Opt. Soc. Am. A 16 (9), (Sep 1999). Pp. 21242135. Avaible

http://pyramid.spd.louisville.edu/ eri/papers_pr es/hass99.pdf

[4] W.K. Pratt. Digital Image Processing. John Wiley \& Sons, Inc., 1978.

[5] Helena C.G. Leitao, Jorge Stolfi. Information contents of fracture lines, Proc. WScG'2000 $8^{\text {th }}$ Intl. Conf. In Central Europe on Computer Graphics etc., Univ. of West Bohemia Press, February, 2000, vol. 2, pp.389-395.

[6] Dong-Keun Kim, Byung-tae Jang, Chi-Jung Hwang. A planar perspective image maching using Point correspondences and rectangle-t0quadrilateral mapping, Fifth IEEE Southwest Symp. on Image Analysis and Interpretation", Apr. 7-9, 2002, Santa Fe, New Mexico. Avaible at

http://www.computer.org/proceedings/ssiai/153 7/15370087abs.htm.

[7] J.A. Withers. Tracking Cell Splits and Merges, Proc. of the IEEE Southwest Symp. on Image Analysis and Interpretation, 1996, pp117-122.
[8] Software MERGEFOUR. Avaible at http://dwww.essc.psu.edu/lasdoc/user/mergefour .html.

[9] Doudkin A.A., Selikhanovich A.M., Sadykhov R. Kh. and Vershok D.A. Contour Extraction Algorithms for LSI Circuit Video Image Processing, Proc. of the International Workshop "Intelligent Data Acquisition and Advanced Computing Systems (IDAACS'2001)”, 2001, Foros, Ukraine, pp. 69-72.

[10] Doudkin A.A., Vershok D.A. ComputerAided Inspection Of Some Topological Rules Of Integrated Circuit Layers. Proc. of the $7^{\text {th }}$ Int. Conf. on Pattern Recognition and Information (PRIP'03). Minsk, 2003, v.1, pp. 92-96.

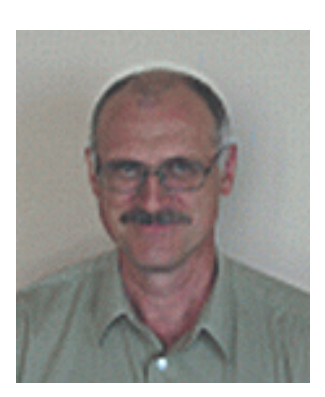

\section{Doudkin Alexander}

Arsent'evich

Education: Graduated from Vitebsk State Pedagogic University in 1972 (diploma with honor).

Academic Qualification: candidate of Sciences degree (an equivalent of Ph.D.) in $C A D$ in 1987, senior researcher (an equivalent of Assistant Professor) in CAD in 1989.

Employment record and duties: PhD student at the Institute of Engineering Cybernetics (IEC), National Academy of Sciences of Belarus (NASB), 1976-1981, junior researcher, researcher, senior researcher at the laboratory of system programming and logic synthesis, IEC, NASB, 1982-1989, senior researcher at the laboratory of VLSI CAD problems, Brest branch of IEC, NASB 1990-1993, leading researcher at the laboratory of systems identification, United Institute of Informatics Problems (UIIP, former IEC),, NASB, 1994 to date.

International activities: chairman of organizing committees of the 5th Int. Conf. on Pattern recognition and Information Processing (PRIP'99), the 2nd and the 3rd Int. Conf. of Neural Networks and Artificial Intelligence (ICNNAI'01, ICNNAI,03), one of the organizers of the PRIP'97, 01 and 03, ICNNAI'99. Member of IAPR, BAPR and the Byelorussian branch of the an international society of neural networks.

Research interests: methods and algorithm for design of digital integrated circuits, computer aided design of control units, digital signal and image processing, pattern recognition, architectures and models of computer vision systems, synthesis of high-performance processors. 


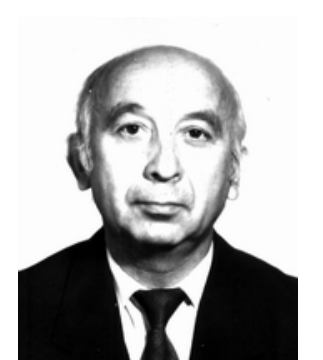

Rauf Kh. Sadykhov, in 1967 graduated from Azerbaijan Polytechnic Institute (Baku) on the specialty "Mathematical and Computing the Instruments and Devices". After graduation from the Institute he attended the postgraduate course at the Institute of Engineering Cybernetics in Minsk. In 1991 he defended his thesis for a scientific degree of a doctor of engineering science in the field of computing science and in 1992 has obtained a professor,s scientific rank.

Since 1995 R.Kh. Sadykhov is a head of Computer System Department in Belarusian State University of Informatics and Radioelectronics and simultaneously he is a head of System Identification laboratory, Institute of Engineering cybernetics of the Belarusian Academy of Sciences. R.Kh. Sadykhov has published more than 350 scientific works, including books, patents, papers and reports at the International Conferences, Symposiums and Workshop. The area of the scientific investigations includes: digital signal and image processing, recognition of handwritten symbol and signature identification, remote-sensing object recognition, computer vision system for the control and recognition, intellectual neural systems, multi-agent systems, parallel architectures for digital signal and image processing.

Prof. R.Sadykhov is the vice-chairman of Belarusian Association of Pattern Recognition (IAPR) and Belarus SIG of International Neural Network Society (INNS), the member of IEE (United Kingdom).

Maksim Vatkin, in 1998 graduated from with honor Brest Polytechnic Institute on specialty "Engineer System Analyst". After graduation form the Institute he attended the postgraduate course at the Institute of Engineering Cybernetics in Minsk. Since 2001 Maksim Vatkin is junior scientist of "Laboratory of System Identification" of "United Institute of Informatics Problems" of the Belarusian National Academy of Science. The area of the scientific investigations includes: artificial intelligence, artificial neural networks, image recognition and analysis. 